\title{
ORIGINAL RESEARCH \\ Radiosurgery for Metastatic Spinal Tumors: Follow-Up MR Findings
}

\author{
Y.J. Hwang \\ M.-J. Sohn \\ B.H. Lee \\ S.Y. Kim \\ J.W. Seo \\ Y.H. Han \\ J.Y. Lee \\ S.J. Cha \\ Y.H. Kim
}

BACKGROUND AND PURPOSE: We reported the MR findings of spinal metastatic lesions after SRS according to the volumetric changes (in press). However, in the case of osteoblastic metastatic lesions, tumor volume usually doesn't change during remission. In this study, we retrospectively analyzed pre- and post-SRS CT and MR imaging findings of locally controlled osteoblastic spinal metastatic lesions besides volumetric changes

MATERIALS AND METHODS: Among 113 spinal metastatic lesions in 72 patients, 10 lesions were osteoblastic. CT and MR images were reviewed to identify 4 parameters: 1) changes in tumor volume, 2) changes in CT attenuation, 3) changes in T2 signal intensities and 4) changes in degrees of contrast enhancement.

RESULTS: Tumor volume was unchanged in 9 lesions and increased in 1 lesion. CT attenuation increased in 7 lesions and T2 signal intensities were heterogenous with dark signal foci within the tumor in 3 of 7 lesions and with dark and bright signal foci within the tumor in 4 of 7 lesions. In 3 lesions where CT attenuation decreased, T2 signal intensity became heterogenous with dark and bright foci in 2 of 3 lesions and T2 signal intensity became normalized in 1 lesion. The degree of contrast enhancement decreased in 8 lesions but 2 lesions showed persistent contrast enhancement.

CONCLUSIONS: Further progression of sclerotic changes was a more common finding than loss of sclerotic foci. Main MR imaging features of osteoblastic lesions after treatment were development of dark with or without bright signal foci, with decrease in contrast enhancement.

ABBREVIATIONS: EBRT $=$ external beam radiation therapy; $S R S=$ stereotactic radiosurgery
$T^{2}$ he spine is the most frequent site of metastatic disease, and approximately $40 \%$ of all cancer patients develop spinal metastases. ${ }^{1}$ The most common symptom of spine involvement is pain, and if the spinal tumor is sufficiently large, weakness and numbness below the level of spinal involvement will develop. Left untreated, many patients become paraplegic or lose control of bowel and bladder function, resulting in significant morbidity and poor quality of life. Early treatment when the patient is still ambulatory improves the chances for a better quality of life. ${ }^{2,3}$

Standard treatment for metastatic spinal tumors includes pain medication, steroids, EBRT, surgical decompression, and chemotherapy. Among these, EBRT is often the initial treatment and achieves varying degrees of pain relief. ${ }^{4,5}$ However, EBRT is limited in affecting only the radiosensitive structures such as the spinal cord. In addition, it must be fractionated into multiple sessions over several weeks to limit toxicity. The radiation dose is also often limited to a level far below the optimal therapeutic dose, resulting in a limited clinical response. $^{6,7}$

In recent years, advances in image-guided navigation have allowed SRS to be applied to spinal and intracranial tumors. A

Received March 25, 2011; accepted after revision May 27.

From the Departments of Radiology (Y.J.H., B.H.L., S.Y.K., J.W.S., Y.H.H., J.Y.L., S.J.C., Y.H.K.) and Neurosurgery (M.-J.S.), Inje University Ilsan Paik Hospital, College of Medicine, Goyang-si, Gyenggi-do, Republic of Korea.

This work was supported by a grant from Inje University in 2005.

Paper was previously presented at the Annual Meeting of the Radiological Society of North America, November 28-December 3, 2010; Chicago, Illinois.

Please address correspondence to Yoon Joon Hwang, Department of Radiology, Inje University IIsan Paik Hospital, College of Medicine, 2240 Daehwa-dong, Ilsanseo-gu, Goyang-si, Gyenggi-do, Republic of Korea; e-mail: hyj@paik.ac.kr

http://dx.doi.org/10.3174/ajnr.A2760 major benefit of SRS is the safe delivery of a large, highly conformal radiation dose to a localized tumor. Precise confinement of the radiation to the target increases the likelihood of tumor control, decreases toxicity to the spinal cord, and allows for a reduction in the number of administered fractions (to 1 to 5). Several studies have demonstrated the technical feasibility and clinical efficacy of SRS for spinal metastatic tumors, and it has become a standard treatment for these tumors. ${ }^{5,8-17}$

The main goals of SRS in this setting are to arrest tumor growth throughout the patient's life (rather than cure the lesions), improve symptoms, and prevent the development or progression of neurologic deficits. Previously published reports about efficacy of SRS for spinal metastatic tumors are focused on just clinical data and outcome.

MR imaging is the primary tool for evaluating spinal tumors and monitoring response to treatment, and volumetric changes are the best marker for the evaluation of the treatment efficacy. However, the other changes of MR imaging findings in metastatic spinal tumors after SRS are not well known, and when the tumor volume is not changed, there is a paucity of information on the status of the tumor. Therefore, we retrospectively analyzed pre- and post-SRS MR imaging findings and evaluated the changes of MR imaging findings according to the volumetric changes of metastatic spinal tumors after SRS.

\section{Materials and Methods}

\section{Patients}

This retrospective study was approved by the institutional review board of our hospital and patient informed consent was waived. Between November 2003 and April 2008, 100 consecutive patients with a total of 180 spinal metastatic tumors were treated with Novalis ra- 
diosurgery at our hospital. All patients had a histologic diagnosis of malignant neoplasm and had either synchronous or metachronous metastasis to the spine. Diagnosis of spinal metastasis was established by typical MR and CT imaging findings, such as paravertebral soft tissue mass, osteolytic or osteoblastic changes, and involvement of posterior elements. Of the 180 lesions, 80 were not subject to follow-up MR imaging after radiosurgery and were excluded from the analyses. Patients who received previous conventional radiation therapy $(n=4)$ or underwent open surgery $(n=14)$ or vertebroplasty ( $n=3$ ) were also excluded. Thus, 44 patients (29 men and 15 women) with 79 tumors were enrolled in this study. Patient age ranged from 28 to 80 years (mean 58.5 years). The 79 spinal metastatic tumors were located in the cervical (5 lesions [6.3\%]), thoracic (35 lesions [44.3\%]), lumbar (32 lesions [40.5\%]), and sacral (7 lesions [8.9\%]) spine. Mean follow-up time was 7.07 months (range 1-29 months). Primary tumor pathology was consistent with hepatocellular carcinoma $(n=12)$, lung cancer $(n=11)$, breast cancer $(n=4)$, prostate cancer $(n=3)$, renal cell cancer $(n=3)$, colon cancer $(n=2)$, nasopharyngeal cancer $(n=2)$, thyroid cancer $(n=2)$, ureter cancer $(n=1)$, parotid cancer $(n=1)$, malignant thymoma $(n=1)$, soft tissue sarcoma $(n=1)$, and squamous cell carcinoma from unknown origin $(n=1)$.

\section{Radiosurgery Technique}

The radiosurgical procedure consisted of 1) image acquisition, 2) stereotactic localization and patient setup, and 3) radiosurgical planning and treatment. For imaging, high-resolution thin-section MR images were obtained (with a 1.5T Symphony; Siemens Medical Systems, Erlangen, Germany). All examinations included both TSE T1weighted (TR, 400-700 ms; TE, $12 \mathrm{~ms}$ ) and TSE T2-weighted (TR, 3000-5000 ms; TE, $108 \mathrm{~ms}$ ) sequences. Before the administration of contrast, imaging of the involved spinal segment (ie, cervical, thoracic, and/or lumbar) was performed with TSE T1- and T2-weighted imaging in the sagittal $(512 \times 256$ matrix with a NEX of 2$)$ and axial $(320 \times 256$ matrix with a NEX of 4$)$ planes. Sagittal imaging was performed with 3-mm sections and a section gap of $1 \mathrm{~mm}$; axial imaging was performed with 2 -mm sections, with no gap and no angles. Intravenous gadolinium was given to all patients $(0.1 \mathrm{mmol} / \mathrm{kg})$. The spine was imaged in the axial and sagittal planes after contrast with TSE T1-weighted sequences. Before CT images were obtained for radiosurgical planning, the patient was brought to a simulating room to prepare the setup of his or her positioning and immobilization in conjunction with the stereotactic devices.

For the stereotactic localization and procedure, the patient was placed in a vacuum bag on an ExacTrac body tray (Brainlab, Feldkirchen, Germany) and immobilized with an aquaplastic mask device. Before the CT scan was performed, the external fiducial markers were affixed to the anterior iliac spine, the skin of the anterior chest wall, and the mask frame. The fiducial skin markers were placed in relation to the locations of the lesions. Then the patient was sent to the CT room under the stereotactic localizer and frame.

The patients were scanned on a 4-detector row CT scanner (Volume Zoom; Siemens Medical Systems). Contrast-enhanced CT images were obtained throughout the range defined by the fiducial markers and stereotactic localizer. The scanning parameters were 110 mAs, 120-140 kVp, FOV $50 \mathrm{~cm}$, collimation $2.5 \mathrm{~mm}$, section thickness $3 \mathrm{~mm}$, and increment $2 \mathrm{~mm}$.

The DICOM data of the MR and CT images were transferred to workstations for radiosurgical planning. To obtain high-resolution spatial information and accurate stereotactic images, the MR images
Table 1: Definitions of 3 groups for volume changes and 4 types of T2 signal changes

Changes

\begin{tabular}{ll}
\hline Group 1 & Decreased in volume \\
Group 2 & Unchanged in volume \\
Group 3 & Increased in volume \\
Type 1 & No changes in T2 signal intensity \\
Type 2 & Increase in T2 signal intensity \\
Type 3 & Increase in T2 signal intensity intermixed with dark signal intensity \\
Type 4 & Changed to totally dark signal intensity \\
\hline
\end{tabular}

were fused, at each vertebral segment, onto the CT images (which included the stereotactic devices) by using the segmental image fusion method. ${ }^{18}$

Radiosurgical teams including a medical physicist and neurosurgeons defined the tumor volume to be irradiated. They then provided optimal dosimetric planning and prescribed a radiation dose and fraction size, which depended on the tumor histology, location, proximity to critical structures, and clinical status. The average dose-fractionation scheme was $35.3 \pm 8.4$ Gy in $1-6$ fractions. Finally, the patient was repositioned with the stereotactic frame and irradiated under image-guided navigation with an infrared tracking system.

\section{Follow-Up MR Imaging Evaluation}

Follow-up MR images were performed with the same parameters as the pretreatment MR images and were retrospectively and independently evaluated by 2 neuroradiologists. These evaluations focused on 1) changes in tumor volume, 2) changes in $T 2$ signal intensity, and 3) changes in contrast enhancement patterns. The tumor volume was measured by performing manual segmentation by using multiple image sections on TSE T1-weighted axial images, with consideration of TSE T1, TSE T2, and contrast-enhanced TSE T1-weighted axial and sagittal images. Volume calculation was then performed by incorporating the data obtained from each of the multiple sections with the assistance of 3D software (Rapidia; Infinitt, Seoul, Korea). All MR images were analyzed in a consensus reading by 2 neuroradiologists (Y.H. and B.L., with 13 and 4 years of experience in spine imaging, respectively). Tumor volume was measured independently by the same neuroradiologists and the mean value was used.

The pre- and posttreatment volume ratios and volumetric changes were used to categorize the tumors as decreased in volume (group 1), unchanged in volume (group 2), and increased in volume (group 3). If the ratio of volumetric changes was within $10 \%$ (the ratio range $0.9-1.1$ ), the lesions were regarded as unchanged in volume.

T2 signal intensity changes of the tumors were categorized into 4 types: type 1 (no changes), type 2 (increase in T2 signal intensity), type 3 (increase in T2 signal intensity intermixed with dark signal intensity), and type 4 (totally dark signal intensity) (Table 1; Fig 1). Enhancement patterns were divided into 2 groups: no change in contrast enhancement and decrease of contrast enhancement with or without nonenhancing foci.

The clinically recommended follow-up schedule was to perform MR imaging during the first 3 months and every 3 months thereafter. Whenever patients had any neurologic signs or aggravation of symptoms, they were asked to come to our institute and additional MR imaging was obtained when needed. The last MR images were used as a comparison with pretreatment images. 

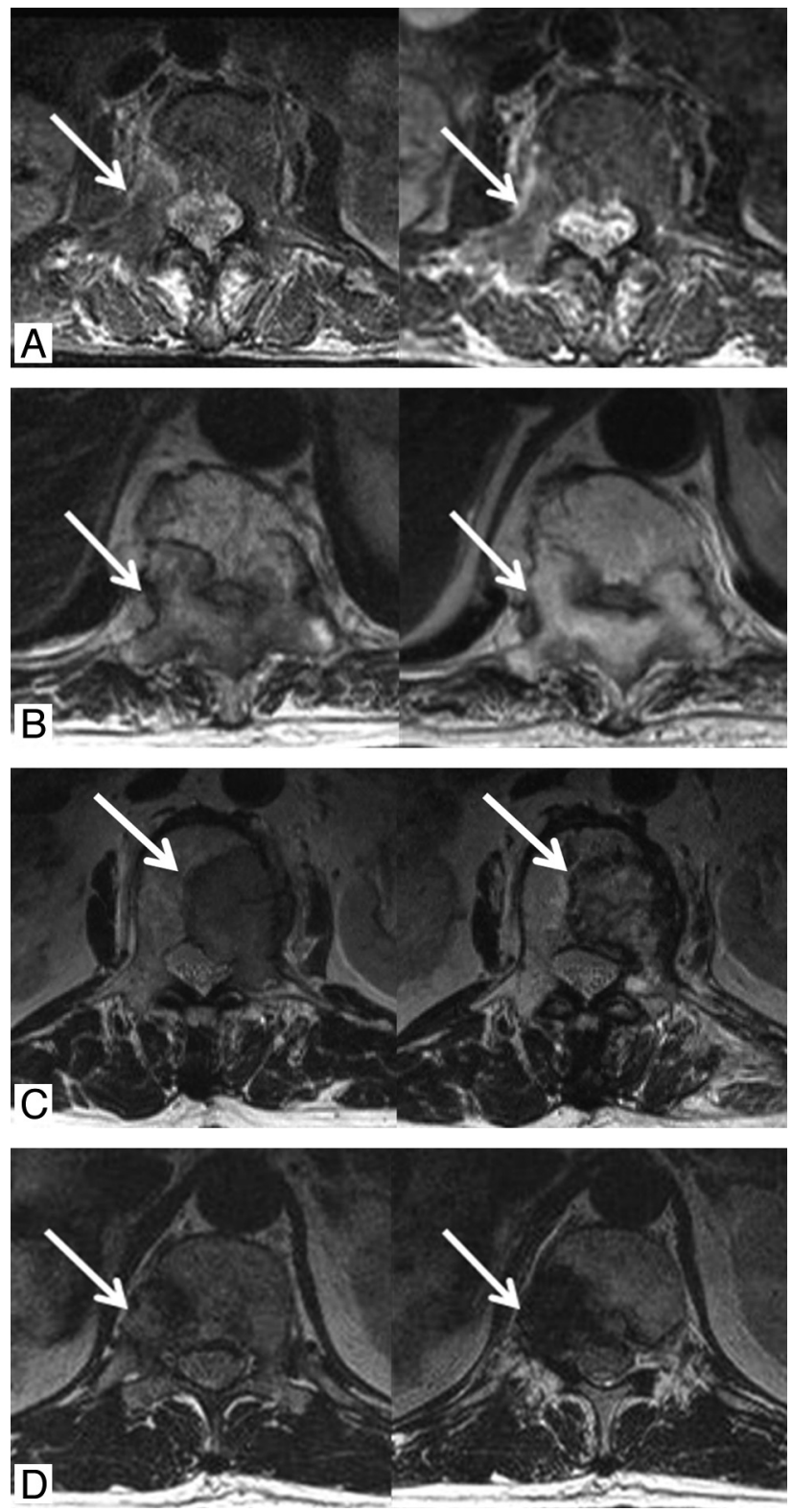

Fig 1. Four types of $\mathrm{T} 2$ signal intensity changes after radiosurgery. A, Type 1: No changes in T2 signal intensity; 67-year-old man with prostate cancer, initial and 3-month follow-up images. $B$, Type 2: Increase homogeneously in T2 signal intensity; 79- year-old woman with thyroid carcinoma, initial and 7-month follow-up images. C, Type 3: Increase in T2 signal intensity with dark signal intensity; 78-year-old woman with colon cancer, initial and 12-month follow-up images. D, Type 4: Totally dark signal intensity; 54-year-old man with lung cancer, initial and 2-month follow-up images.

\section{Results}

\section{Changes in Tumor Volume}

The volumetric changes of each group are summarized in Table 2. The mean initial pretreatment tumor volume was $40,006 \mathrm{~mm}^{3}$ (range $510-423,401 \mathrm{~mm}^{3}$ ). Among the $79 \mathrm{le}-$ sions, 32 (40.5\%) decreased in volume (group 1), 39 (49.4\%) remained unchanged (group 2), and $8(10.1 \%)$ increased in volume (group 3). Thus, 73 (group $1+$ group 2 [89.9\%]) of 79 lesions were stable or decreased in size and were thus considered locally controlled.

The mean follow-up periods for groups 1, 2, and 3, were

\begin{tabular}{lccc}
\hline \multicolumn{4}{c}{ Table 2: Volumetric changes of each group } \\
\hline & $\begin{array}{c}\text { Pretreatment } \\
\text { Volume } \\
\left(\mathrm{mm}^{3}\right)\end{array}$ & $\begin{array}{c}\text { Posttreatment } \\
\text { Volume } \\
\left(\mathrm{mm}^{3}\right)\end{array}$ & Ratio \\
\hline & $64,742.39$ & $42,750.41$ & 0.65 \\
Group 1 $(n=32)$ & $(1274-423,401)$ & $(441-257,075)$ & $(0.08-0.88)$ \\
& $19,906.46$ & $19,337.26$ & 0.98 \\
Group 2 $(n=39)$ & $(510-112,890)$ & $(515-120,200)$ & $(0.9-1.08)$ \\
& $39,051.75$ & $54,334.00$ & 1.60 \\
Group 3 $(n=8)$ & $(1292-136,909)$ & $(1357-169,066)$ & $(1.17-2.82)$ \\
& & &
\end{tabular}

6.69 months (range 1-27 months), 6.54 months (range 1-29 months), and 5.5 months (range 1-11 months), respectively.

\section{Changes in T2 Signal Intensity and Contrast Enhancement Patterns}

Table 3 summarizes the changes in T2 signal intensity and contrast enhancement patterns. In group 1, the most common findings were increased T2 signal intensity intermixed with dark signal intensity (type 3), and $28(87.5 \%)$ of the total 32 lesions were seen with this pattern (Fig 2 ). The signal intensity on T2-weighted images increased homogeneously in 2 lesions (6.25\%; type 2) and changed to dark (type 4 ) in the other 2 lesions $(6.25 \%)$. Contrast enhancement decreased and nonenhancing foci developed in 31 lesions (96.9\%). Only 1 lesion $(3.1 \%)$ showed persistent homogeneous enhancement and this lesion was of type 3 .

In group 2, the most common findings were also type 3 $(36 / 39,92.3 \%)$. The other 3 lesions were categorized into type $1(2 / 39,5.1 \%)$ and type $2(1 / 39,2.6 \%)$, and contrast enhancement persisted in these 3 lesions.

In group 3 (8 lesions), 2 (25.0\%) type 4 lesions changed to homogeneous dark signal intensity without contrast enhancement (Fig 3). In 2 lesions (25\%), there were no significant interval changes in T2 signal intensity and the degree of contrast enhancement and categorized type 1 (Fig 4). In 4 type 3 lesions, contrast enhancement decreased and the initial tumor volume also decreased, but local recurrences developed at the margins of the lesions and the total volume of the tumors increased (Fig 5).

We also classified metastatic lesions into osteolytic, mixed, and osteoblastic based on the CT images used for stereotactic localization. Among the 79 lesions, 60 lesions were osteolytic, 14 mixed, and 5 osteoblastic. Four of 5 lesions showed type 3 patterns, and 1 lesion showed type 4 pattern. Four type 3 lesions were unchanged in volume, but 1 type 4 lesion, in the area of the T1 signal intensity changes, was increased and categorized in group 3. Contrast enhancements were decreased in all osteoblastic lesions.

\section{Discussion}

The development of advanced image-guided techniques has made possible the delivery of large doses of radiation to very tightly defined targets. These techniques have increased the chance of local control of spinal metastatic tumors, with SRS emerging as a new treatment option. Several studies on the efficacy of SRS for spinal metastatic tumors have been reported recently. ${ }^{5,8-17}$ Although MR imaging is the best tool to evaluate the status of metastatic spinal tumors, many results about treatment efficacy are confined to clinical data such as 


\begin{tabular}{|c|c|c|c|c|c|c|}
\hline & \multicolumn{2}{|c|}{$\begin{array}{c}\text { Group } 1(n=32) \\
\text { Contrast Enhancement }\end{array}$} & \multicolumn{2}{|c|}{$\begin{array}{c}\text { Group } 2(n=39) \\
\text { Contrast Enhancement }\end{array}$} & \multicolumn{2}{|c|}{$\begin{array}{c}\text { Group } 3(n=8) \\
\text { Contrast Enhancement }\end{array}$} \\
\hline & Decreased & Persistent & Decreased & Persistent & Decreased & Persistent \\
\hline Type $1(n=4)$ & 0 & 0 & 0 & 2 & 0 & 2 \\
\hline Type $2(n=3)$ & 2 & 0 & 0 & 1 & 0 & 0 \\
\hline Type 3 ( $n=68)$ & 27 & 1 & 36 & 0 & 4 & 0 \\
\hline Type $4(n=4)$ & 2 & 0 & 0 & 0 & 2 & 0 \\
\hline
\end{tabular}
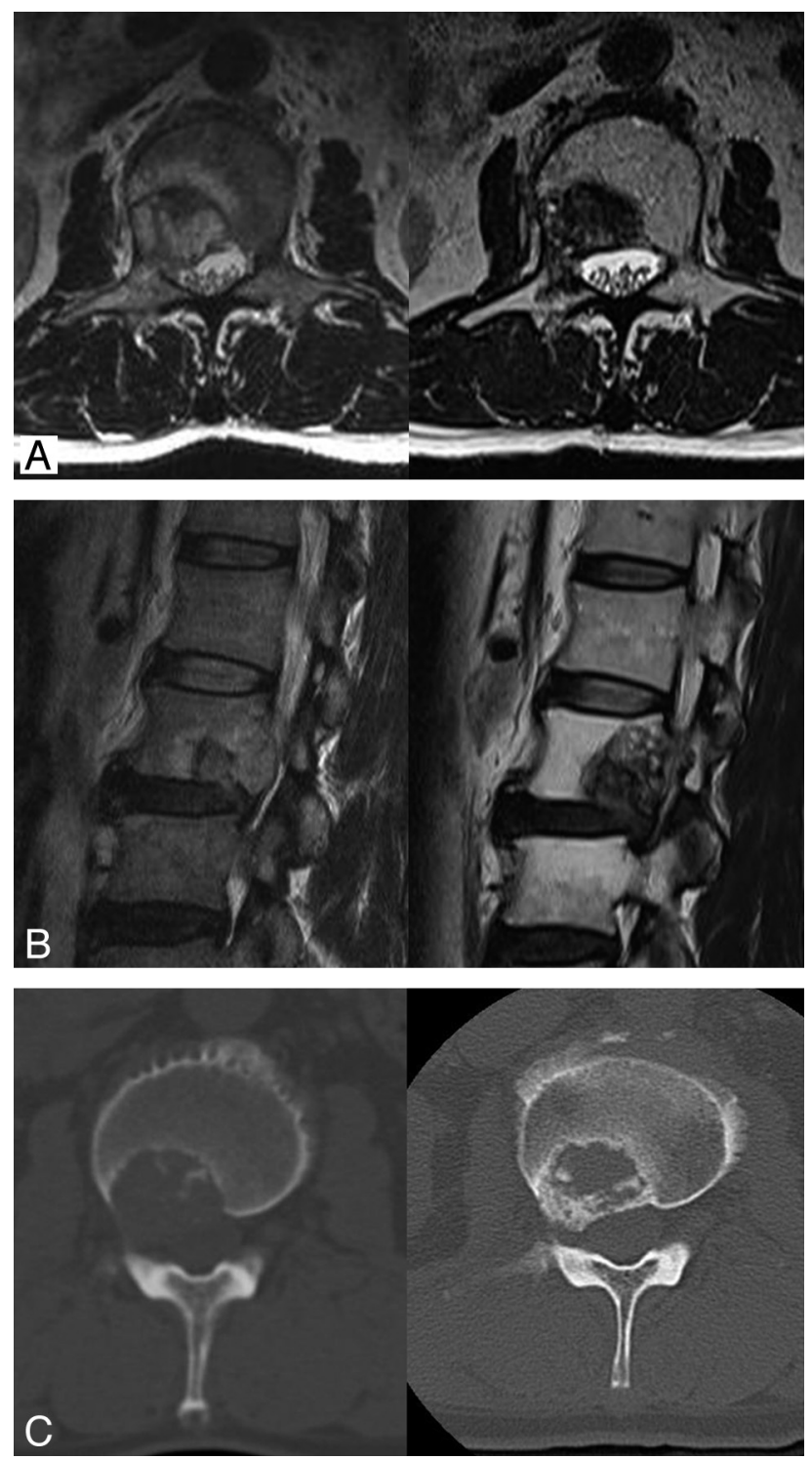

Fig 2. Initial and 27-month follow-up MR and CT images in 52-year-old man with lung cancer. $A$ and $B$, T2 signal intensities of metastatic lesion in L3 body and right pedicle increased and intermixed with dark signal intensity on T2-weighted axial and sagittal images. $C$, Sclerotic changes are seen on follow-up CT image.

pain control. . $^{8,10,11,17}$ Some reports have included measurements of tumor volume by MR imaging but have not provided detailed MR imaging findings. ${ }^{12,13,15,16}$ The life span of affected patients is limited, in most cases, by systemic disease. As a result, patients and clinicians tend to focus on systemic problems rather than spinal metastases, unless the latter is causing pain or neurologic deficits. Thus, follow-up MR imaging is often not performed after the initial treatment of spinal me-
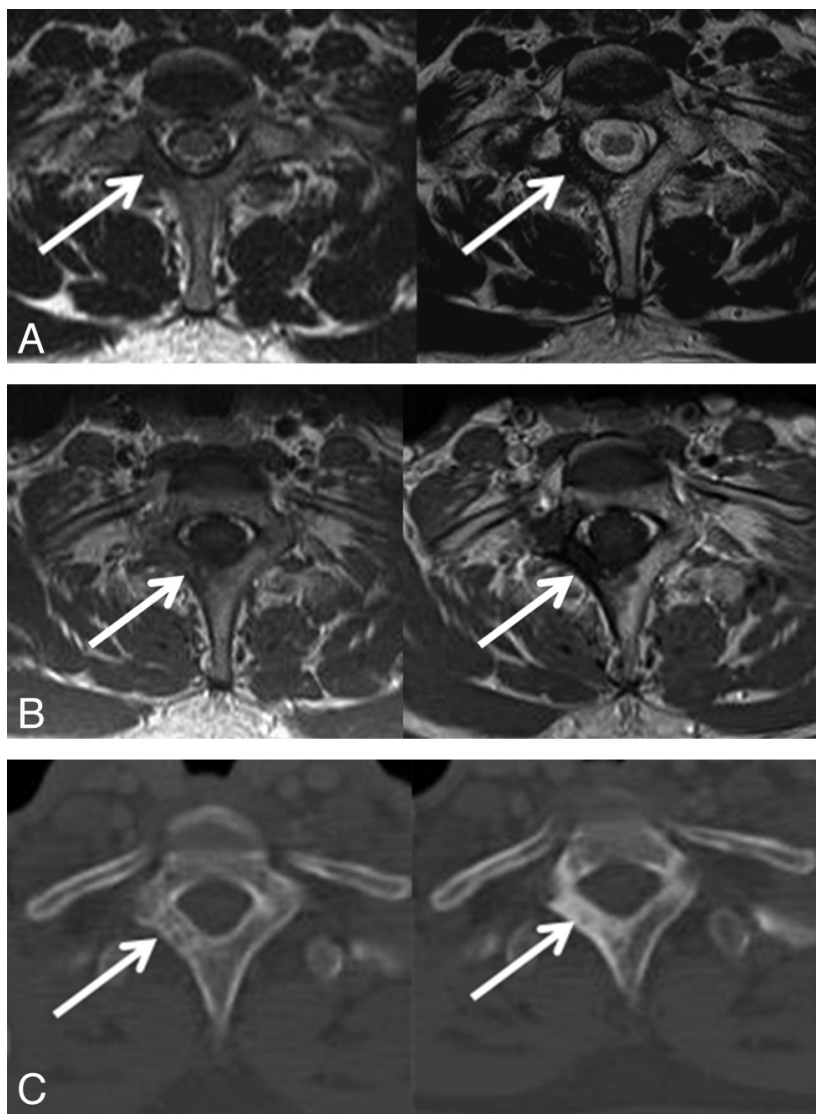

Fig 3. Expanded appearance with progressed sclerotic change. Initial and 11-month follow-up MR and CT images in 47-year-old woman with breast cancer. A, Signal intensity of metastatic lesion changed to dark signal intensity and area of signal intensity changes seemed to be increased on T2-weighted axial image. $B$, Contrast enhancement was not seen on contrast-enhanced T1 axial images. $C, 0$ n CT image, sclerotic changes progressed at the periphery of the lesion.

tastases because it is not considered a priority or because the patient's condition is too poor or unstable. However, an accurate follow-up evaluation of a spinal metastatic tumor may benefit clinical management, as one can detect tumor recurrences or compressive deformities and then administer an appropriate treatment, such as boost radiation therapy.

In our study, variable signal intensity changes and enhancement patterns were observed, and the most common findings were increased T2 signal intensity, combined with dark signal intensity changes (type 3), and stable or decreased tumor volume (groups 1 and 2). These were considered indicative of local tumor control. Pollen and Shlaer ${ }^{19}$ described osteoblastic response on plain radiographs after successful treatment, including chemotherapy of metastatic cancer of the prostate. Others ${ }^{20-22}$ also reported that development of a sclerotic rim at the periphery of the tumors, followed by centrip- 

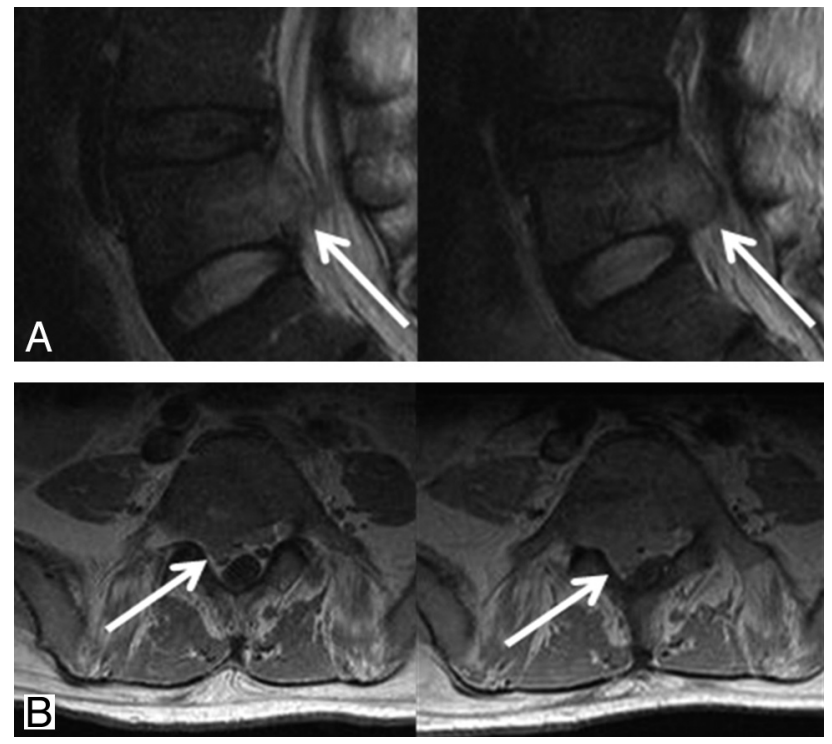

Fig 4. Tumor growth without changes in $\mathrm{T} 2$ signal intensity and contrast enhancement pattern. Initial and 2-month follow-up MR images in 47-year-old man with hepatocellular carcinoma. $A$, T2 signal intensity shows no significant interval change on follow-up T2 sagittal image. $B$, There was also no significant interval change in contrast enhancement pattern on contrast-enhanced T1-weighted axial image, but tumor volume slightly increased with compression of dural sac.

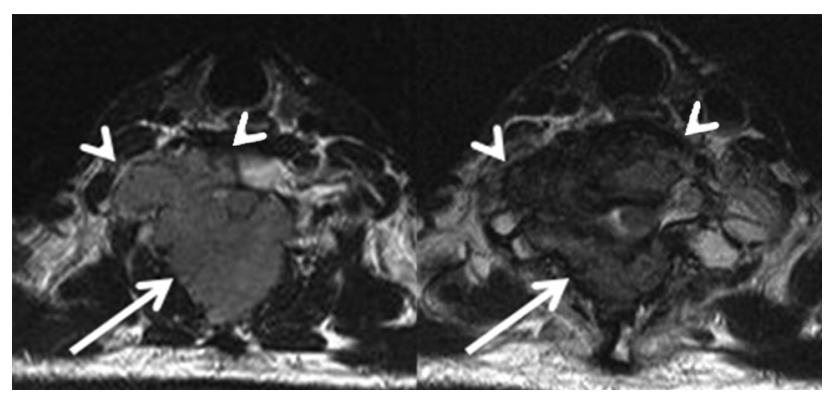

Fig 5. Initial local control, with the subsequent growth of new lesions at the periphery of the tumors. Initial and 8-month follow-up T2 axial images in a 47-year-old man with hepatocellular carcinoma. Tumor volume in posterior compartment was decreased (arrow), but the tumor grew at the periphery of spinal body (arrowhead).

etal filling of the whole lytic area, is the best sign of a healing response in the radiographs of osteolytic metastatic lesions. These sclerotic rims and osteoblastic changes in the radiographs are thought to be concordant with the dark signal intensities on T2-weighted images (Fig 2).

T2 signal intensities were increased in $89.9 \%$ (types 2 and 3) of all lesions and mainly considered the result of tumor necrosis. But Sanchez et $\mathrm{al}^{23}$ correlated pathologic specimens of primary musculoskeletal neoplasms after chemotherapy with MR images, and reported that areas of high signal intensity on T2WI corresponded to areas of residual viable tumor, tumor necrosis, edema, fibrosis, and hemorrhage. In our study, decreased but persistent contrast enhancement was observed in the area of increased T2 signal intensity. We think that viable tumor tissue was still present between the necrotic tissues and that the stronger, more homogenous contrast enhancement is proportional to a higher recurrence rate.

In 4 type 4 lesions, T2 signal intensity became totally dark, and we considered these a more advanced sclerotic form of the type 3 lesions. Contrast enhancement was not present in these lesions, and they seemed completely locally controlled. However, in 2 lesions, as sclerotic changes progressed at the periphery, the total area of dark signal intensity changes increased and the final volume seemed to increase (Fig 3). This expanded appearance has been described on plain films ${ }^{20,24}$ and should not be misunderstood as tumor progression.

Persistent contrast enhancements were seen in all 4 type 1 lesions (100\%) and in 1 of 3 type 2 lesions (33.3\%). The rate of persistent contrast enhancement was significantly higher in types 1 and 2 lesions than in types 3 ( 1 of 69 [1.5\%]) and 4 $(0 \%)$ lesions. This difference suggests that tumor cells in types 1 and 2 lesions have a greater potential to remain viable. Thus, lesions that are classified as type 1 or 2 warrant closer clinical observation, especially given their higher recurrence rate.

Among the 8 lesions in group 3, actual tumor growth was considered present in 6; the other 2 lesions showed homogeneous T2 dark signal intensity with expanded appearance. Two patterns of tumor growth were identified: 1) growth without changes in T2 signal intensity and contrast enhancement (Fig 4), and 2) initial local control, with the subsequent growth of new lesions at the periphery of the tumors during follow-up (Fig 5). Two of the 6 group 3 lesions thought to have tumor growth showed the first pattern, and this was thought to result from an insufficient radiation dosage or radioresistance in the tumor. The other 4 lesions in this group of 6 showed a typically favorable response in the early stage of treatment, but the residual viable tumor tissues seemed to have grown at the tumor margin, causing an increase in the final tumor volume. If the patients had survived longer and undergone additional follow-up imaging, this pattern of recurrence would probably have been more prevalent.

We evaluated the osteoblastic lesions separately. Among these, the most common T2 signal intensity changes were also type 3, and all but 1 lesion showed no change in tumor volume. The 1 enlarged tumor showed expanded appearance with totally dark signal intensity changes. This result is not different from the result of entire lesions, but the number of cases is very small and further additional evaluations about osteoblastic lesions will be needed.

There were several limitations in this study. First, our study was retrospective. We agreed on the fact that preplanned sequential MR imaging follow-up studies are helpful to manage clinical symptoms or control tumor progression, but practically, follow-up MR imaging has been ordered based on clinical change. Patients with spinal metastatic lesions often had a poor general condition such that follow-up imaging was often not performed due to systemic disease. As a result, the follow-up period varied from 1 to 29 months and evaluations were performed at different intervals. We evaluated only the last MR image. This would be the second limitation, and a serial evaluation would be more helpful to observe the pattern of changes after treatment. Third, a variety of different tumors were treated. But in our study, the response and imaging appearance could not be evaluated in each type of tumor because the numbers of each tumor were so small. Fourth, we were not able to compare the radiologic analysis with clinical parameters and radiologic-pathologic correlation was not performed. 


\section{Conclusions}

The most common MR imaging findings after stereotactic radiosurgery of spinal metastatic tumors were increased T2 signal intensity intermixed with T2 dark signal intensity, suggesting sclerotic changes, and decreased contrast enhancement. When T2 signal intensity shows no changes, or increased signal intensity without dark signal intensities, close follow-up is recommended. Patterns of tumor growth were classified as indicative of either 1) volume increase without changes of signal intensity and contrast enhancement, or 2) initial local control, but growth, at the periphery of the tumor during followup. Although volumetric changes of the metastatic tumors after SRS is the best marker for response, correlation with T2 signal intensity changes and contrast enhancement patterns can give additional information for the current status of spinal metastatic lesions after treatment.

\section{References}

1. Wong DA, Fornasier VL, MacNab I. Spinal metastases: the obvious, the occult, and the impostors. Spine 1990;15:1-4

2. Black P. Spinal metastasis: current status and recommended guidelines for management. Neurosurgery 1979;5:726-46

3. Helweg-Larsen S. Clinical outcome in metastatic spinal cord compression. A prospective study of 153 patients. Acta Neurol Scand 1996;94:269-75

4. Wu Js, Wong R, Johnston M, et al. Cancer Care Ontario Practice Guidelines Initiative Supportive Care Group. Meta-analysis of dose-fractionated radiotherapy trials for the palliation of painful bone metastases. Int J Radiat Oncol Biol Phys 2003;55:594-605

5. Gerszten PC, Mendel E, Yamada Y. Radiotherapy and radiosurgery for metastatic spine disease. What are the options, indications, and outcomes? Spine 2009;34:S78-92

6. Faul CM, Flickinger JC. The use of radiation in the management of spinal metastases. J Neurooncol 1995;23:149-61

7. Ryu SI, Chang SD, Kim DH, et al. Image-guided hypo-fractionated stereotactic radiosurgery to spinal lesions. Neurosurgery 2001;49:838-46

8. Ryu S, Yin FF, Rock J, et al. Image-guided and intensity-modulated radiosurgery for patients with spinal metastasis. Cancer 2003;97:2013-18
9. Rock JP, Ryu S, Yin FF. Novalis radiosurgery for metastatic spine tumors. Neurosurg Clin N Am 2004;15:503-09

10. Degen JW, Gagnon GJ, Voyadzis JM, et al. CyberKnife stereotactic radiosurgical treatment of spinal tumors for pain control and quality of life. J Neurosurg Spine 2005;2:540-49

11. Muacevic A, Staehler M, Drexler C, et al. Technical description, phantom accuracy, and clinical feasibility for fiducial-free frameless real-time imageguided spinal radiosurgery. J Neurosurg Spine 2006;5:303-12

12. Gerszten PC, Burton SA, Ozhasoglu C, et al. Radiosurgery for spinal metastases: clinical experience in $\mathbf{5 0 0}$ cases from a single institution. Spine 2007;32:193-99

13. Chang EL, Shiu AS, Mendel E, et al. Phase I/II study of stereotactic body radiotherapy for spinal metastasis and its pattern of failure. J Neurosurg Spine 2007;7:151-60

14. Sahgal A, Larson DA, Chang EL. Stereotactic body radiosurgery for spinal metastases: a critical review. Int J Radiat Oncol Biol Phys 2008;71:652-65

15. Sheehan JP, Shaffrey CI, Schlesinger D, et al. Radiosurgery in the treatment of spinal metastases: tumor control, survival, and quality of life after helical tomotherapy. Neurosurgery 2009;65:1052-61

16. Tsai JT, Lin JW, Chiu WT, et al. Assessment of image-guided CyberKnife radiosurgery for metastatic spine tumors. J Neurooncol 2009;94:119-27

17. Gagnon GJ, Nasr NM, Liao JJ, et al. Treatment of spinal tumors using cyberknife fractionated stereotactic radiosurgery: pain and quality-of-life assessment after treatment in $\mathbf{2 0 0}$ patients. Neurosurgery 2009;64:297-306, discussion 306-07

18. Sohn MJ, Lee DJ, Yoon SW, et al. The effective application of segmental image fusion in spinal radiosurgery for improved targeting of spinal tumours. Acto Neurochir 2009;151:231-38

19. Pollen JJ, Shlaer WJ. Osteoblastic response to successful treatment of metastatic cancer of the prostate. AJR Am J Roentgenol 1979;132:927-31

20. Barry WF Jr, Wells SA Jr, Cox CE, et al. Clinical and radiographic correlations in breast cancer patients with osseous metastases. Skeletal Radiol 1981;6:27-32

21. Libshitz HI, Hortobagyi GN. Radiographic evaluation of therapeutic response in bony metastases of breast cancer. Skeletal Radiol 1981;7:159-65

22. Hortobagyi GN, Libshitz HI, Seabold JE. Osseous metastases of breast cancer. Clinical, biochemical, radiographic, and scintigraphic evaluation of response to therapy. Cancer 1984;53:577-82

23. Sanchez RB, Quinn SF, Walling A, et al. Musculoskeletal neoplasms after intraarterial chemotherapy: correlation of MR images with pathologic specimens. Radiology 1990;174:237-40

24. Pagani JJ, Libshitz HI. Imaging bone metastases. Radiol Clin North Am 1982; 20:545-60 


\section{Erratum}

The article "Radiosurgery for Metastatic Spinal Tumors: Follow-Up MR Findings" (2012;33:382-87) contained an incorrect abstract. The authors regret this error. The correct abstract is reproduced below:

Background and Purpose: MR imaging is the primary tool for evaluation and monitoring of spinal tumors. We retrospectively analyzed the MR imaging findings before and after SRS for metastatic spinal tumors.

Materials and Methods: We reviewed MR imaging findings on 79 metastatic spinal tumor lesions in 44 patients (29 male and 15 female) who had undergone radiosurgery between November 2003 and April 2008. Posttreatment MR imaging was evaluated retrospectively for 3 aspects: 1) changes in tumor volume; 2) changes in T2 signal intensity; and 3 ) changes in contrast enhancement patterns.

Results: With regard to tumor volume on MR images, 32 lesions $(40.5 \%)$ decreased in volume (group 1), 39 (49.4\%) showed no change (group 2), and 8 (10.1\%) increased in volume (group 3). T2 signal intensities were unchanged in 4 lesions (type 1), homogeneously increased in 3 (type 2), and changed to a homogeneously dark signal in 4 (type 4). The T2 signal intensity was increased and intermixed with dark signal intensity (type 3 ) in 68 lesions. A decrease in contrast enhancement with or without non-enhancing foci was seen in 73 lesions. A persistent homogeneous enhancement pattern was seen in all 4 of the type 1 lesions, in 1 of the 3 type 2 lesions, and in 1 of the 68 type 3 lesions.

Conclusions: Main MR imaging features of locally controlled metastatic spinal tumors included no increase in tumor volume, increased T2 signal intensity with intermixed T2 dark signal intensity, and decreased contrast enhancement. Follow-up MR imaging also provided several patterns of tumor recurrence.

http://dx.doi.org/10.3174/ajnr.A3131 